\title{
A Mixed-methods Exploration of Young Adults' Views of the Sexual Double Standard
}

\author{
Charlotte Wesson $^{1}$ (D)
}

Accepted: 6 January 2022 / Published online: 28 February 2022

(c) The Author(s) 2022

\begin{abstract}
The traditional sexual double standard occurs when men and women are evaluated differently for the same sexual behaviours. Typically, women are judged more punitively than their male counterparts regarding promiscuous behaviour. There is much research surrounding this double standard. However, there has been less investigation into what factors individuals consider responsible for this phenomenon. The present study aimed to use qualitative and quantitative methods to examine young adults' attitudes to this double standard. Three main themes were observed and are discussed across this article. These themes include, Theme (1) Attitudes from the past, Theme (2) (Un)acceptability of behaviour and Theme (3) Media portrayal.
\end{abstract}

Keywords Sexual double standard $\cdot$ Sexuality $\cdot$ Gender $\cdot$ Attitudes

\section{Introduction}

The Sexual Double Standard (SDS) is a phenomenon in which men and women are viewed differently for engaging in comparable sexual behaviours. This manifests with men usually being praised, while women are stigmatised (Bordini \& Sperb, 2013).

Two theories as to why the SDS occurs include (I) evolutionary theory and (II) social role theory. Evolutionary theory postulates that the SDS arises because of evolutionary adaptations, one of which concerns mate selection. Traditionally, women play a greater role in child rearing as compared to men, whose role is over after successful fertilisation (Zaikman \& Marks, 2017). Additionally, women can theoretically have fewer children than men due to carrying the pregnancy, thus women

Charlotte Wesson

char.wesson@gmail.com

1 School of Psychology, University of Lincoln, Lincoln, UK 
must ensure the survival of their offspring. Due to these differences, women benefit more from long-term mates, who they can screen for genetic quality and resources for their offspring. In contrast, men do not have such evolutionary concern (Zaikman $\&$ Marks, 2017). This links with the SDS because people may attribute positive personality characteristics to those displaying signs of reproductive fitness, and negative traits to those demonstrating signs of reproductive weaknesses. For instance, high levels of female sexual activity can lead to paternity uncertainty (Zaikman \& Marks, 2017).

The second theory is social role theory. This postulates that the SDS occurs because society expects different behaviours from men and women, which in part is due to physical differences between men and women, e.g., men are physically stronger (Zaikman \& Marks, 2017). Traditionally, men's activities involved more dominant behaviour (e.g., permission to have multiple sexual partners), whereas women's activities were more submissive (e.g., being a homemaker). Thus, men are praised for being sexually active, whereas women are not. It is presumed that adherence to, or violation of, gender roles contribute to the SDS phenomenon (Zaikman \& Marks, 2017).

Though there is much debate surrounding whether this double standard still exists in modern society (e.g., Bordini \& Sperb, 2013), there is less evidence examining what individuals believe is responsible for it. Continuing to investigate the SDS is important for many reasons. Critically, it can impact women's safety. Women may be placed at risk of 'undesired consequences' if sexual expectations are not met (Zaikman \& Marks, 2014), as men allegedly report higher expectations for post-date sexual activity if it is the woman who instigates the date (Morr Serewicz \& Gale, 2008). For instance, Jozkowski et al. (2017) found that more promiscuous men believed they could 'convince' women who 'passively refused' sex to change their mind. Moreover, understanding the SDS is necessary for women's sexual well-being. It is thought that the SDS affects women's sexual autonomy. Crawford and Popp (2003) suggest women exchange said autonomy for social desirability. This means that they may they refuse sex even when they are willing to engage in it due to the SDS. Thus, they are potentially becoming disconnected from their own desires. Likewise, Sanchez et al. (2012) suggest that adhering to stereotypical sexual scripts (i.e., female 'submission') affects women's sexual autonomy. Crucially, women may internalise this 'submissive' role, automatically linking 'sex' with 'submission'. Evidently, the SDS can have a significant impact on women both as a sociocultural collective and at individual level. Therefore, it is important to investigate what contributes to this phenomenon.

This study aimed to examine young adults' (aged 18 to 25) views of the SDS (i.e., whether they believed it exists and what is responsible for it). Young adults were selected as the target population because this group is undergoing an important period of continuing development, in terms of sexual identity and relationship formation. This transitional period in an individual's life also has implications for negative outcomes relating to sexuality (e.g., engaging in risky sexual behaviours), as well as positive outcomes (e.g., sexual self-efficacy and self-esteem; Boislard et al., 2016). Thus, it is important to explore young adults' views of the SDS and why they believe it occurs to inform educational materials. 


\section{Method}

A mixed methods approach was used. SDS research usually relies on vignettes detailing hypothetical situations/individuals. This technique can limit the ecological validity of studies and to what extent the SDS operates in the real world (Marks et al., 2018). It has been said that qualitative studies demonstrate a clearer picture of the SDS compared to quantitative studies (Marks et al., 2018).

Ethical approval was gained through University of Lincoln's ethical board (SOPREC) as part of a wider PhD study (approval code: PSY1415134).

\section{Participants}

A total of 63 participants took part in this study, including 12 heterosexual men, two homosexual men, two bisexual men, 37 heterosexual women, one homosexual female and nine bisexual women, aged between 18 and $25(M=19.60, S D=1.53)$.

\section{Materials}

Participants were given a paper questionnaire pack which consisted of a demographic questions (i.e., gender, age, sexuality); and the 'Sexual Double Standard Scale' (SDSS; Muehlenhard \& Quackenbush, 1998). At the end of the questionnaire pack, there were also some questions relating to the SDS. These included:

'I believe that a 'double standard' exists whereby it is more acceptable for a man to have multiple sexual partners/casual sex, than it is for a woman to exhibit the same behaviour'-participants were required to rate their agreement from 1 (strongly agree) to 5 (strongly disagree) where 3 was 'undecided'.

'What do you believe is responsible for this double standard?' - this was left as an open-ended response for participants to complete.

\section{Procedure}

Participants were recruited via opportunity sampling, primarily from the University's online recruitment system (SONA). They reported to the Psychology department laboratories at University of Lincoln. It is of note that this study was part of a larger $\mathrm{PhD}$ study looking at measurement of sexual interest using approach-avoidance measures. Thus, the order of the questionnaire (analysed in this study) and the experimental methods were counterbalanced between-subjects. Participants were presented with a brief about the study and fully informed consent was obtained. Participants were made aware that they were able to withdraw consent and participation in the study at any point before, during, or up to two weeks after participation, without reason. Once participants had completed the study, they were fully debriefed and were given a debrief form to take away with them. 


\section{Details of Data Analysis}

Quantitative data were analysed in IBM SPSS Statistics 25. Qualitative data were analysed through reflexive thematic analysis, following recommendations and guidance by Braun and Clarke (2006). Data were analysed in an inductive framework, as this is grounded in the data itself; and identified at a semantic level, examining only that which has been written; and finally within an essentialist/realist method, focusing on participants' experiences and reality (Braun \& Clarke, 2006). Qualitative data were subject to initial coding, leading to a total of 20 initial codes. These codes were then sorted into data-driven themes, which were then further refined and reviewed into the final themes and subthemes presented here. Specific details for initial codes, initial themes, and final themes are presented in (see Appendix A, B, and $\mathrm{C}$, respectively).

\section{Results}

Scores for the SDSS can range from 48 (greater acceptance of sexual freedom for men) to -30 (greater acceptance of sexual freedom for women), where 0 is equivocal acceptance. For men, scores ranged from 1 to $16(M=7.19, S D=3.94)$ and for women, scores ranged from -4 to $25(M=5.47, S D=4.51)$. Interpreting these results, men showed greater acceptance of male (compared to female) sexual freedom compared to women. However, an independent samples t-test showed that scores did not differ significantly between men and women $(t(59)=1.35, p=.181)$. However, when looking at Cohen's $d$ scores, a small-to-medium effect size was observed $(d=0.41)$. Moreover, it is of interest to note that, in absolute terms, women did not show scores that indicate markedly more acceptance of sexual freedom for women. A one-sample t-test indicated that scores differed significantly from 0 (equivocal acceptance) for men $(t(15)=7.30, p<.001)$ and women $(t(44)=8.13, p<.001)$. When looking at the means ( 7.19 and 5.47 for men and women, respectively), this suggests that both men and women differ from equivocal acceptance in the direction of greater acceptance of sexual freedom for men.

Fig. 1 Response (split by gender) indicating whether participants believe a sexual double standard exists

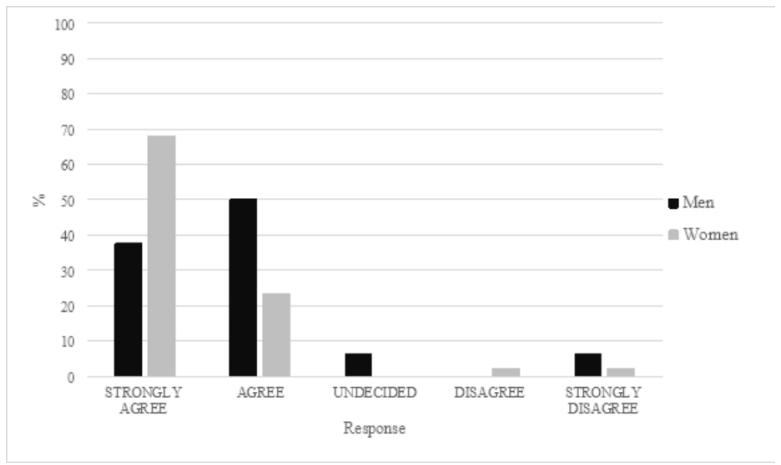


Figure 1 depicts participant response to the question 'I believe that a 'double standard' exists whereby it is more acceptable for a man to have multiple sexual partners/ casual sex, than it is for a woman to exhibit the same behaviour'. As can be seen from this graph, a greater number of women compared to men strongly agreed with this statement, with the majority of men only agreeing. Notably, more men (relative to women) strongly disagreed with this statement.

\section{Qualitative Data}

The themes identified were, Theme (1) Attitudes from the past; Theme (2) (Un) acceptability of behaviour; and Theme (3) Media portrayal. Sub-themes were also identified, which will be discussed below. Participants will be referred to by a specific code e.g., Participant number 1 who is a woman and 20 -years-old will be represented as $[1, \mathrm{~F} 20]$.

Theme 1: Attitudes from the past. A prominent theme across this data set was that attitudes from the past are responsible for the SDS. Many of the answers received were framed in the past tense, rather than the present tense. For instance, individuals referred to "historical ideals", "historic views", "old fashioned" ideas and things as being in or from "the past". For instance, responses included: "the attitudes of older generations" [26,M18]; "ancestor views and values" [24,M21]; "different era" [22,M21]; "older opinions"[61,F19]; "old fashioned ideas about the social roles of women/men"[59,F20]; "historically women weren't allowed to do such things, I think it's a matter of past society" [41,F19]; and "society's attitudes towards sex from the past" [21,F19]. It appears that some individuals blame the attitudes of past generations for current SDS, rather than seeing it as an issue formed from attitudes in today's society. It is indeed important to acknowledge that past attitudes have shaped the SDS. However, it is equally important to recognise that the SDS still exists in present society (Bordini \& Sperb, 2013).

One sub-theme recognised within 'Attitudes from the past', is 'religiosity'. Though religion still plays a part in modern society, this is often less prominent than it once was. Typically, religious views are still seen as more conservative and less permissive of certain sexual acts or free expression of one's sexuality. Thus, such perceptions may explain why some individuals referenced 'Attitudes from the past' as a contributing factor to SDS. One facet of religiosity that may have contributed to this viewpoint is that men have been treated more favourably in religion than women. For instance, while polygamy is practised by multiple religions, its occurrence comprises almost exclusively of polygyny (one husband with multiple wives; Brooks, 2009). Some participants just referenced the umbrella terms "religion" [16,F18] [45,M19]; "religious belief" [56,F20][26,M18]; or "religious influences" [26,M18], as being responsible for the SDS. However, others refer more indirectly to religion in their responses. For instance, "belief that a woman's virginity is sacred" [4,F19]. Moreover, individuals often referenced the need for women to be 'pure'. For example, "women are meant to be viewed as "pure" [3,F19]; "women should remain "pure"'[48,F20]; "society's views of women that they should stay "pure" [1,F19] and "need for women to be 'pure"'[9,F19]. The Collins dictionary defines 'pure' as "a person, especially a woman, who is described as pure is considered to be morally 
good, especially because they have no sexual experience" as well as "free from sin" (Collins, 2019). This definition likely plays a part in participants interpretation of religion contributing to the SDS. This also potentially makes it difficult to challenge the SDS, as it is ingrained even in the terminology that is used in modern day society.

A second sub-theme identified in terms of 'Attitudes to the past' is 'traditional gender roles'. Traditional gender roles include the idea that men possess more power than women. Similarly, traditional gender roles establish men as being careeroriented, whilst women are associated with domestic duties (Zaikman \& Marks, 2014). The former of these attitudes was suggested by participants in the current study. For example: "girl/woman as more 'vulnerable' than a man/boy"[39,F22]; "men are seen as more dominant"[54,F19]; "history of men being more powerful than women"[20,F18]; "dependency of women on men"[32,F20]; "historical ideals regarding women's role as the submissive"[9,F19]. Within the sub-theme of 'traditional gender roles' some participants also referenced the objectification of women. Quotes include, "women as nothing more than sexual objects"[30,F19] and "view as 'object' for a man to use as he wants" [31,F19]. These statements suggest that women are perceived as not having their own agency and are only an extension of a man or object a man possesses, rather than being their own being with their own free-will and desires (including sexual desires). This means that women are potentially being viewed as an object that men have sex with, but it is not plausible that women would have their own sex life.

Theme 2: (Un)acceptability of behaviour. The second key theme from the current data set involves what participants believed society considers (un)acceptable behaviour for men and women. This theme was not necessarily limited to sexual behaviour. Some individuals did not refer to either gender in a pejorative manner, e.g., "social constructs of acceptable sexual activity across genders" [49,M18] and "stereotypes of how males and females should behave" [57,F18]. This theme linked to the prior discussed sub-theme of 'traditional gender roles'. However, some participants did specify a gender orientation as to (Un)acceptability of behaviour. Generally, women are thought to be judged more punitively than men. Examples include, "society's views [...] that a promiscuous woman is less desirable" [1,F19] and "women are supposed to act lady like and in a respectful manner" [40,F20]. Similarly, it was observed that society was more permissive towards men. For example, "it is considered the cultural norm for men" [38,F19]; "people believe men should only have casual sex" $[33, F 20]$; and "[...] more traditional views whereby men could get away with more" $[48, F 20]$. This demonstrates how young adults are aware that it is the differential treatment of the different genders that perpetuates the SDS.

Two sub-themes are linked to (Un)acceptability of behaviour. The first is 'male reputation'. Generally, male reputation is thought to be enhanced by multiple sexual partners and/or casual encounters. Men are often praised for such activities, e.g., "guys get a pat on the back when they sleep with someone new"[3,F19]; "men are seen as 'cool' for having multiple sexual partners"[54,F19]; and "modern society telling men sleeping with lots of women makes them 'cool"'[37,F19]. Similarly, some individuals referred to 'lad' culture, e.g., "lad culture" [30,F19] [60,M20] and "stereotypes of guys going out drinking and being a 'lad' for having one night stands" [46,F21]. 'Lad culture' encompasses behaviours relating to excessive alcohol con- 
sumption, sport, and 'banter' (Phipps \& Young, 2013), as well as 'pack behaviour', misogyny, and objectification of women (Jeffries, 2020). It has also been suggested that 'lad culture' is aligned with toxic masculinity (Diaz-Fernandex \& Evans, 2020). This suggests a particularly ingrained issue within society, that is not only damaging to women but also to men themselves.

A related, but distinct, sub-theme identified is 'uncontrolled biological factors'. Typically, participants just referred to "biology" [40,F20]; "men's sexual drive" [25,F20] "evolution" [14,M22][16,F18]; "evolutionary processes" [52,M25]; and "biological differences between males and females" [58,M20]. However, one participant described this theme in more depth, "men are more sexual than women, therefore are more easily excused when expressing more sexual behaviours" [63,F24]. Participants suggest that the perceived biological differences between men and women contribute to the SDS, in that men are 'designed' to be liberal with their sexuality, whilst women are reproductively more selective.

Theme 3: Media portrayal. The final theme is media portrayal referring to the differing depiction of men and women in mainstream media. Though this was referenced multiple times across the current data set, most individuals simply referenced "the media" without much elaboration [11,M18][12,F23][16,F18][34,F19][39,F22] [47,M20][50,M19] [52,M25][56,F20]. Generally, it seemed that media portrayal was linked to previously mentioned themes. One example is "Media-women are always portrayed as the sub always needing protecting and staying pure, whilst me are strong and have to conquer, etc." [10,F20]. Another example is "the media and the idea of a girl/woman as more 'vulnerable' than a man/boy" [39,F22]. As is demonstrated here, these examples encompass one or more of the previous themes/sub-themes. Both of these suggest that mainstream media plays a large role in perpetuating the stereotypical roles of men and women. However, without further elaboration from participants, it is difficult to explain exactly what is meant by media portrayal in this sample. However, one individual did state "media portrays it [as] more acceptable for a man" $[23, F 20]$. It can only be assumed that the 'it' that is being referred to is men having multiple sexual partners/casual sex. Zaikman and Marks (2017) suggested that mainstream media "bombard young people with images and portrayals of female sexuality as dirty, dangerous, taboo, and male sexuality as normal, proper, or good" (p. 416). Moreover, Shafer et al., (2013) state that traditional sexual scripts in the media depict men 'actively and aggressively' pursuing women and sex, with these women characters being judged by their sexual conduct. This gives some clarity to this theme. As nearly a quarter of participants in this study referred to the media as being wholly or partly responsible for the SDS, it is imperative to consider this moving forward. For instance, what is portrayed in the media and inevitably consumed by young adults may shape their views on the SDS, further perpetuating it. In their theoretical paper, Zaikman and Marks (2017) suggested that, as the media usually portrays societal ideals of men and women (including sexual behaviour), individuals who are heavy media consumers will be more likely to endorse a double standard than those who consume less media. This could be of particular concern for young adults, who are known for (sometimes excessive) media consumption (Shafer et al., 2013). 


\section{Discussion}

This study aimed to identify whether young adults believe an SDS exists, and what they believe is responsible for this phenomenon. Both quantitative and qualitative (thematic) analysis was used. Quantitative data suggested that men showed greater acceptance of male (compared to female) sexual freedom compared to women. Though this difference was statistically non-significant, Cohen's $d$ scores did reveal a small-to-medium effect size when looking at the differences between men's and women's scores. This suggests that this difference is likely not statistically significant as the sample is lacking in power, therefore it may be the case that if the sample were larger, a statistically significant difference would be found. Also, the majority of participants indicated that they believed a sexual double standard exists. Qualitative data revealed three main themes as to what young adults believe is responsible for the SDS: Attitudes from the past, (Un)acceptability of behaviour, and Media portrayal. Many of the responses were constructed in relation to the past, i.e., it is older generations who have enforced these attitudes, rather than them being perpetuated by the present generation. Sub-themes relating to Attitudes from the past include traditional gender roles (as seen in the past) and religiosity (which was highly influential in the past). The first sub-theme of religiosity suggested that women should be less promiscuous as preserving their sexuality is more important than men's, due to women providing life. Crawford and Popp (2003) acknowledged that women are either seen as pure and virginal, or promiscuous and easy, there is no in between. It appears that sexuality, and thus virginity, is confused for morality, where women are seen as less valued if this virginity is not 'intact' (d'Avignon, 2016). Moreover, a women's intact virginity is advantageous for men as it ensures certainty of paternity (as seen in the evolutionary hypothesis; d'Avignon, 2016). The second sub-theme of traditional gender roles gives support to the social role theory of SDS. In recent years there has been a movement away from traditional gender roles. The National Centre for Social Research (2017) found that $72 \%$ of people surveyed disagreed with men needing to be career-oriented and women domestic. However, society still has a long way to go in order to significantly change the impact of these roles on other views, such as the SDS.

The second theme related to what society believes is acceptable behaviour for men and women. This was not inherently sexual. Other behaviours were referenced in terms of etiquette. Notably, these references were mainly directed towards women (e.g., "behaving in a ladylike manner"; "being submissive"). Participants typically expressed that casual sex was perceived as more acceptable for men. Specifically, they suggested that a man's reputation is actually enhanced by promiscuity. Whilst individuals often referenced older generations' views as influencing the SDS, some referred to 'lad culture', which is a newer creation of modern society that involves sexism and other objectifying behaviours (Phipps \& Young, 2015). A concerning aspect of this is the sub-theme of 'uncontrolled biological factors'. Participants suggested that it was biological differences between men and women that resulted in the double standard. This lends itself to the evolutionary theory of the SDS. The explanation of the SDS being caused by perceived biological differences could be particularly damaging. This is because biological factors are commonly perceived as uncontrol- 
lable. Thus, it poses the question of how can the SDS be addressed if one of the contributing factors is 'out of our hands'? This view then leads to the perpetuation of the SDS as people will simply accept that it is a 'fact of life'. Moreover, this biological standpoint suggests that men are less accountable for their actions (e.g., having casual encounters) compared with women, as they are biologically 'programmed' to do this. The acceptance of biological determinism contributing to the SDS is a major issue in the prevention of the SDS and associated negative behaviours. Biological factors are not uncontrollable or necessarily deterministic. Biological-deterministic explanations can be notably reductionist on their own (Ellison \& De Wet, 2018), and sexual selection in humans is actually far more complicated than women being the 'choosy' sex and men being the 'indiscriminate' sex (Gowaty, 2018). Thus, though there may be inherent biological differences between men and women, this does not mean that individuals do not have conscious control over their subsequent actions. In sum "biological determinism does not account for the role of context and culture in the production of persons" (Greene, 2004, p.433).

In conclusion, this research helps to advance the understanding of what young adults believe is responsible for the SDS. Though it is apparent that the role of the SDS may be changing in society, it is still somewhat present. One prominent factor that this study has found to contribute to the SDS is media portrayal, with nearly a quarter of participants referencing the media as a contributing factor for the SDS. Therefore, the media could be an effective tool to attempt to address the SDS in a way that young adults' will be receptive to, if it does indeed exist.

\section{Appendix A-Initial Codes}

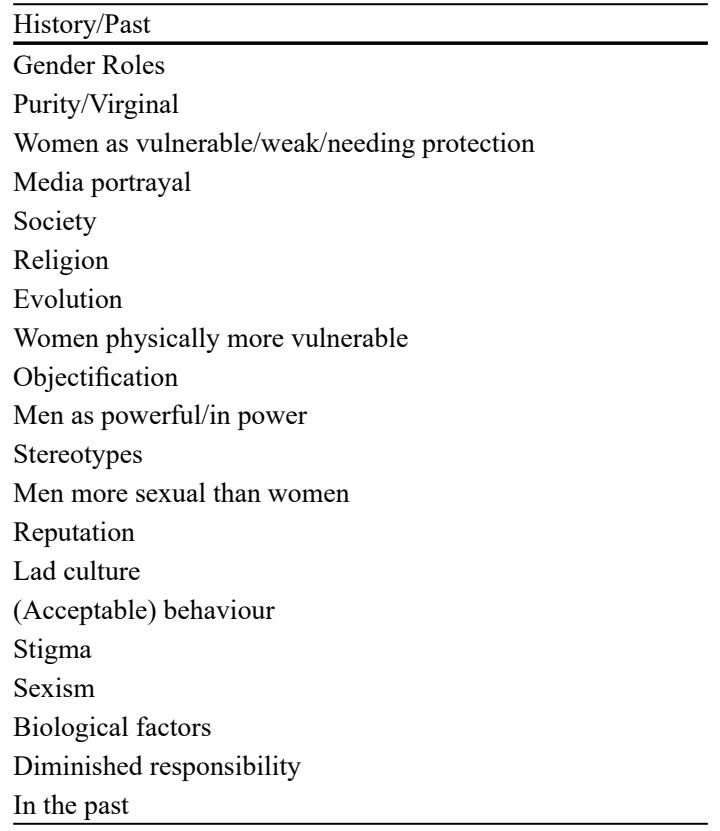




\section{Appendix B-Initial Theme Map}

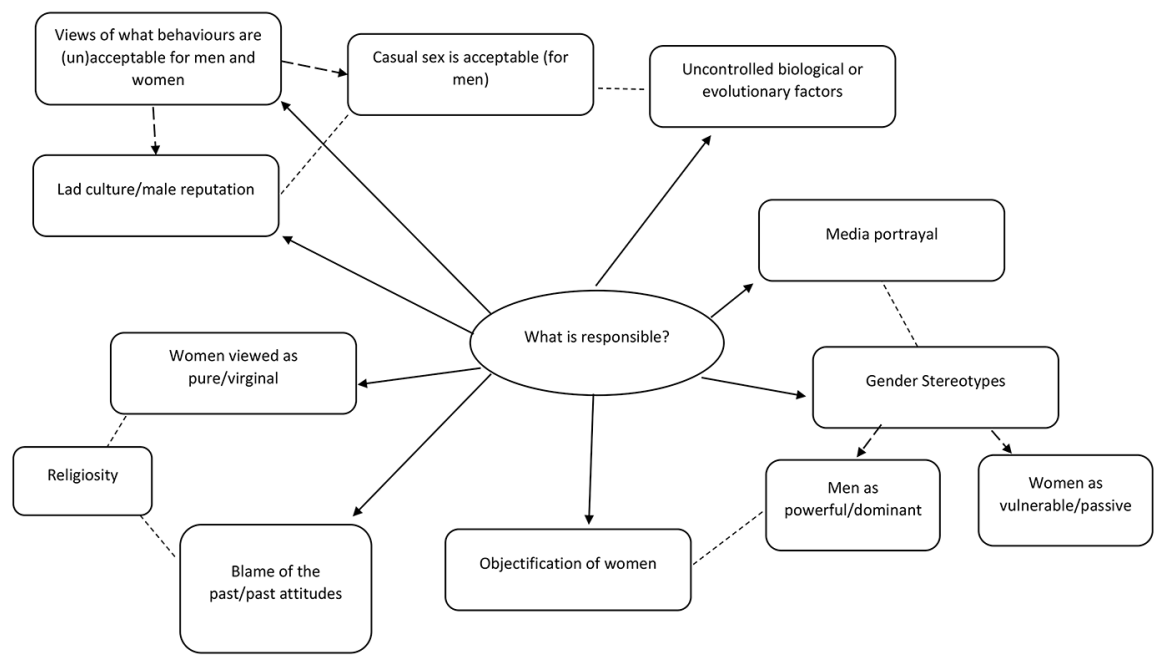

\section{Appendix C_Final Theme Map}

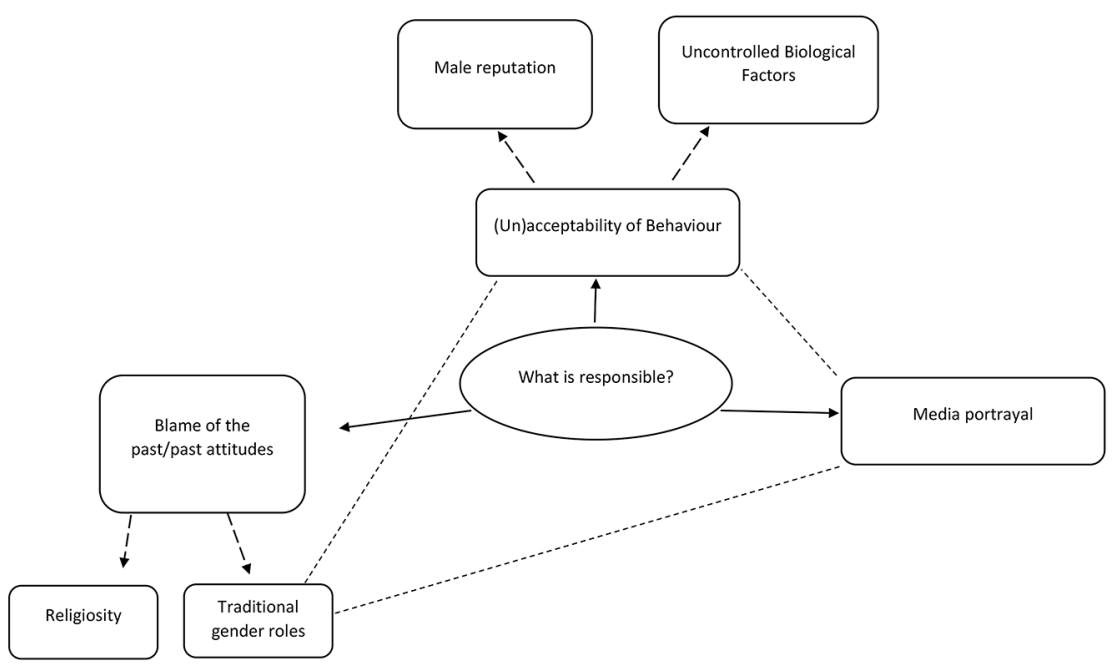

Funding No funding was received for conducting this study.

Availability of Data and Material Data set is available from here: https://osf.io/grm4x. 


\section{Declarations}

Conflicts of Interest/Competing Interests The author has no relevant financial or non-financial interested to disclose.

Ethics approval Ethical approval was gained from University of Lincoln's ethical board (SOPREC) as part of a wider PhD study (approval code: PSY1415134).

Informed Consent Fully informed consent was gained from all participants.

Open Access This article is licensed under a Creative Commons Attribution 4.0 International License, which permits use, sharing, adaptation, distribution and reproduction in any medium or format, as long as you give appropriate credit to the original author(s) and the source, provide a link to the Creative Commons licence, and indicate if changes were made. The images or other third party material in this article are included in the article's Creative Commons licence, unless indicated otherwise in a credit line to the material. If material is not included in the article's Creative Commons licence and your intended use is not permitted by statutory regulation or exceeds the permitted use, you will need to obtain permission directly from the copyright holder. To view a copy of this licence, visit http:/creativecommons.org/ licenses/by/4.0/.

\section{References}

Boislard, M. A., van de Bongardt, D., \& Blais, M. (2016). Sexuality (and lack thereof) adolescence and early adulthood: A review of the literature. Behavioral Sciences, 6(1), 8-32. DOI: https://doi. org/10.3390/bs6010008

Bordini, G. S., \& Sperb, T. M. (2013). Sexual double standard: A review of the literature between 2001 and 2010. Sexuality \& Culture, 17(4), 686-704. DOI: https://doi.org/10.1007/s12119-012-9163-0

Braun, V., \& Clarke, V. (2006). Using thematic analysis in psychology. Qualitative research in psychology, $3(2), 77-101$

Brooks, T. (2009). The problem with polygamy. Philosophical Topics, 37(2), 109-122. DOI: https://doi. org/10.5840/philtopics20093727.

Collins (2019). Collins Dictionary: Definition of 'Pure'. Retrieved from https://www.collinsdictionary. com/dictionary/english/pure

Crawford, M., \& Popp, D. (2003). Sexual double standards: A review and methodological critique of two decades of research. Journal of sex research, 40(1), 13-26. DOI: https://doi. org/10.1080/00224490309552163

d'Avignon, A. (2016). Why have we always been so obsessed with virginity? [Web log post]. Retrieved from https://medium.com/the-establishment/a-quick-and-dirty-history-of-virginity-9ceb24b7e08a

Ellison, G. \& De Wet (2018). Biological Determinism. In Trevethan, W. (Ed.), The International Encyclopedia of Biological Anthropology (pp. 1-4). Wiley

Gowaty, P. (2018). Biological Essentialism, Gender, True Belief, Confirmation Biases, and Skepticism. In Travis, C. B., \& White, J. W. (Eds.), APA Handbook of the Psychology of Women: Vol. 1 History, Theory, and Battlegrounds (pp. 145-164). American Psychological Association

Greene, S. (2004). Biological Determinism: Persisting Problems for the Psychology of Women. Feminism \& Psychology, 14(3), 431-435. DOI: https://doi.org/10.1177/0959-353504044648.

Jeffries, M. (2020). 'Is it okay to go out on the pull without it being nasty?': lads' performance of lad culture. Gender and Education, 32(7), 908-925. DOI: https://doi.org/10.1080/09540253.2019.1594706.

Jozkowski, K. N., Marcantonio, T. L., \& Hunt, M. E. (2017). College students' sexual consent communication and perceptions of sexual double standards: A qualitative investigation. Perspectives on sexual and reproductive health, 49(4), 237-244. DOI: https://doi.org/10.1363/psrh.12041

Marks, M. J., Young, T. M., \& Zaikman, Y. (2018). The Sexual Double Standard in the Real World. Social Psychology. DOI: https://doi.org/10.1027/1864-9335/a000362

Morr Serewicz, M. C., \& Gale, E. (2008). First-date scripts: Gender roles, context, and relationship. Sex Roles, 58(3-4), 149-164. DOI: https://doi.org/10.1007/s11199-007-9283-4. 
Muehlenhard, C. L., \& Quackenbush, D. M. (1998). Sexual double standard scale [Measurement instrument]. Routledge

The National Centre for Social Research (2017). British Social Attitudes. Retrieved from http://bsa.natcen. ac.uk/media/39248/bsa35_gender.pdf

Phipps, A., \& Young, I. (2015). 'Lad culture' in higher education: Agency in the sexualization debates. Sexualities, 18(4), 459-479. DOI: https://doi.org/10.1177/1363460714550909.

Sanchez, D. T., Fetterolf, J. C., \& Rudman, L. A. (2012). Eroticizing inequality in the United States: The consequences and determinants of traditional gender role adherence in intimate relationships. Journal of Sex Research, 49(2-3), 168-183. DOI: https://doi.org/10.1080/00224499.2011.653699

Shafer, A., Bobkowski, P., \& Brown, J. D. (2013). Sexual media practice: How adolescents select, engage with, and are affected by sexual media. In Dill, K. E. (Ed.), The Oxford handbook of media psychology (pp. 223-251). Oxford University Press

Zaikman, Y., \& Marks, M. J. (2014). Ambivalent sexism and the sexual double standard. Sex Roles, 71(910), 333-344. DOI: https://doi.org/10.1007/s11199-014-0417-1

Zaikman, Y., \& Marks, M. J. (2017). Promoting theory-based perspectives in sexual double standard research. Sex Roles, 76(7-8), 407-420. DOI: https://doi.org/10.1007/s11199-016-0677-z

Publisher's Note Springer Nature remains neutral with regard to jurisdictional claims in published maps and institutional affiliations. 